Published in final edited form as:

J Pain. 2013 December ; 14(12 0): . doi:10.1016/j.jpain.2013.07.018.

\title{
Clinical orofacial characteristics associated with risk of first- onset TMD: the OPPERA prospective cohort study
}

\author{
Richard Ohrbach, \\ Department of Oral Diagnostic Sciences University at Buffalo \\ Eric Bair, \\ Departments of Endodontics and Biostatistics University of North Carolina-Chapel Hill
}

Roger B. Fillingim,

University of Florida, College of Dentistry

Yoly Gonzalez,

Department of Oral Diagnostic Sciences University at Buffalo

Sharon M. Gordon,

Department of Oral-Maxillofacial Surgery and Brotman Facial Pain Center University of Maryland School of Dentistry

Pei-Feng Lim,

University of North Carolina-Chapel Hill

Margarete Ribeiro-Dasilva,

Department of Restorative Dental Sciences - Division of Prosthodontics University of Florida, College of Dentistry, Gainesville, FI 32610, USA

Luda Diatchenko,

University of North Carolina-Chapel Hill

Ron Dubner,

Department of Neural and Pain Sciences University of Maryland School of Dentistry

Joel D. Greenspan,

Department of Neural and Pain Sciences, and Brotman Facial Pain Center University of Maryland School of Dentistry

Charles Knott,

Center for Analytics and Public Health Battelle Memorial Institute

William Maixner,

University of North Carolina-Chapel Hill

(C) 2013 The American Pain Society. Published by Elsevier Inc. All rights reserved.

Correspondence to: Richard Ohrbach, DDS PhD Department of Oral Diagnostic Sciences University at Buffalo Buffalo NY 14214 Voice: 716-829-3590 ohrbach@buffalo.edu.

Publisher's Disclaimer: This is a PDF file of an unedited manuscript that has been accepted for publication. As a service to our customers we are providing this early version of the manuscript. The manuscript will undergo copyediting, typesetting, and review of the resulting proof before it is published in its final citable form. Please note that during the production process errors may be discovered which could affect the content, and all legal disclaimers that apply to the journal pertain.

DISCLOSURES

Other authors declare no competing interests.

Portions of these data were presented at the 2012 Annual Scientific Meeting of the International Association for Dental Research in Iguaçu Falls, Brazil. 
Shad Smith, and

University of North Carolina-Chapel Hill

\title{
Gary Slade
}

University of North Carolina-Chapel Hill

\begin{abstract}
Case-control studies have documented clinical manifestations of chronic temporomandibular disorders (TMD), whereas clinical predictors of TMD development are largely unknown. We evaluated 41 clinical orofacial characteristics thought to predict first-onset TMD in a prospective cohort study of U.S. adults aged 18-44 years. During the median 2.8-year follow-up period, 2,737 people completed quarterly screening questionnaires. Those reporting symptoms were examined and 260 people were identified with first-onset TMD. Univariate and multivariate Cox regression models quantified associations between baseline clinical orofacial measures and TMD incidence. Significant predictors from baseline self-report instruments included oral parafunctions, prior facial pain and its life-impact, TMJ noises and jaw locking, and non-specific orofacial symptoms. Significant predictors from the baseline clinical examination were pain on jaw opening and pain from palpation of masticatory, neck, and body muscles. Examiner assessments of TMJ noise and tooth wear facets did not predict incidence. In multivariate analysis, non-specific orofacial symptoms, pain from jaw opening and oral parafunctions predicted TMD incidence. The results indicate that only a few orofacial examination findings influenced TMD incidence, and only to a modest degree. More pronounced influences were found for self-reported symptoms, particularly those that appeared to reflect alterations to systems beyond the masticatory tissues.
\end{abstract}

\section{Keywords}

temporomandibular disorders; cohort studies; trauma; parafunction; pain

\section{INTRODUCTION}

Temporomandibular disorders (TMD) comprise a set of conditions affecting the masticatory muscles or joints. Diagnosis criteria include limited jaw movement, problems of noises or locking in the temporomandibular joint (TMJ), and pain that is aggravated by jaw function. ${ }^{40}$ Pain, however, is the main symptom driving treatment-seeking.

The literature depicts two contrasting etiologic models of acute TMD. In one model, clinical symptoms are consequences of dysregulation in systems beyond the masticatory tissues, such as the central nervous system (CNS) ${ }^{26}$ Pain in the masticatory system is a primary manifestation, and limited jaw movement and TMJ problems are consequences. In the other model, oral parafunctional behaviors or trauma cause damage to masticatory tissues; peripheral nociceptive changes are a consequence of that damage, contributing to pain and functional limitation. A variation of the latter model proposes that masticatory tissue damage sufficient to cause nociception is caused by dental or structural abnormalities but the evidence to date is not supportive for these as primary or even contributing factors for either nociception or TMD. ${ }^{11-13,18,45,47}$ The processes in both models are plausible; moreover, the contrast between them likely represents a false dichotomy. One possibility is that bidirectional effects occur between masticatory tissue damage and pain regulatory systems. Furthermore, one model might explain initial TMD development, while the other might account for TMD worsening and chronicity. The OPPERA heuristic ${ }^{24}$ attempts to integrate the two models by depicting processes involved in acute and chronic TMD: nociceptive musculoskeletal pain begins with peripheral input, and peripheral sensitization soon occurs as a normal part of the nociceptive system's protective role; for some individuals, chronicity 
occurs in the form of neuroplasticity, central sensitization, and cortical reorganization, which is augmented by the role that psychological and behavioral factors take in affecting the pain state.

Prospective cohort studies of initially TMD-free individuals create the best opportunity to evaluate the two models. With this design, putative risk factors measured prior to onset of TMD establish a temporal sequence linking a putative cause to subsequent case development. This temporal sequence is a primary criterion for causal inference.

The OPPERA project (Orofacial Pain: Prospective Evaluation and Risk Assessment) used a prospective cohort study design to investigate risk factors for first-onset TMD, proposing contributions from two primary domains: CNS-regulated pain amplification and psychological distress. ${ }^{24} \mathrm{~A}$ third environmental domain was proposed in which jaw injury, parafunction and other clinically-assessed, pre-clinical signs and symptoms of jaw pain or dysfunction might contribute to TMD risk or interact with the two primary domains. The aim of this paper was to characterize the contributions of those clinical characteristics to the risk of developing TMD. A related aim was to identify underlying domains of clinical orofacial signs and symptoms from among the large number of items evaluated in a comprehensive examination for TMD.

\section{METHODS}

\section{Recruitment, Eligibility Criteria, and Enrollment}

This paper reports findings from the OPPERA prospective cohort study of 2,737 people who were enrolled in 2006-08 and followed for up to 5.2 years in order to identify those who developed temporomandibular disorder (TMD). At enrollment, the sample of communitybased volunteers at the four study U.S. sites was aged 18-44 years and did not have painful TMD when examined using modified Research Diagnostic Criteria for TMD (RDC/ TMD). ${ }^{14}$ At enrollment, study participants also completed questionnaires, autonomic function and sensitivity to sensory stimuli were evaluated, and a blood sample was collected for genotyping. This paper focuses on contributions of baseline measurements of the masticatory system to subsequent risk of developing TMD. Additional methodological detail is available elsewhere in this volume. ${ }^{2}$

\section{Ethical Conduct of Research with Humans}

Study participants provided informed, signed consent to participate in the study. The OPPERA project was reviewed and approved by institutional review boards at each of the study sites and at the data coordinating center, Battelle Memorial Institute.

\section{Study Measures}

A previous publication describes full details of the baseline measures including copies of questionnaires. ${ }^{32}$ For these data, the summary descriptions of the baseline measures are organized into three sets of putative risk factors.

Self-reported putative etiologic factors-Lifetime history of regional trauma was assessed using a check-list of five potentially traumatic experiences (see CPSQ Q33, Appendix $\mathrm{A}^{32}$ ). Lifetime history of injury by yawning and by prolonged mouth opening (CPSQ Q34), and history of orthodontic procedures (CPSQ Q35) were assessed with binary variables. 
Parafunctional behaviors were reported using the Oral Behaviors Checklist which yields a single scale representing the frequency of 21 activities such as clenching, chewing gum, and holding objects between the teeth. ${ }^{25,31}$

Clinical status by self-report-Pain and disability due to orofacial pain was measured using the Grade Chronic Pain Scale. ${ }^{51,52}$ Participants also reported the number of days that their efficiency had been reduced to less than $50 \%$ due to orofacial pain (CPSQ Q12A).

Modifying factors: Factors making orofacial pain better or worse were assessed with a 5item checklist (CPSQ Q8), and an ordinal summary measure was computed.

Limitations in using the jaw were reported with the Jaw Functional Limitation Scale (JFLS) which yields three limitation sub-scales: mastication, vertical jaw mobility, and verbal and emotional expression. ${ }^{33,35}$ Reliability of the instrument is 0.87 (Cronbach's alpha) and 0.87 (temporal stability), while validity, as assessed via known groups comparison, is excellent. A total score was also computed from the three subscales when all three component scores were available.

Orofacial symptoms that were not primarily painful (hereafter: "non-specific orofacial symptoms") were evaluated in relation to the TMJ or masticatory muscle area using a checklist (CPSQ Q1) that asked about six symptoms in the preceding month: jaw stiffness, cramping, fatigue, pressure, soreness, and ache.

TMJ clicking and locking: Experiences of TMJ noises, pain with those noises, and jaw locking were assessed for the past month and for the period prior to the past month (CPSQ Q17-19, Q21, Q25, Q27, Q30).

Clinical status by examination-Examiners, trained according to RDC/TMD specifications, ${ }^{14}$ collected clinical measures using physical assessments and structured interviews to determine whether mandibular mobility and palpation produced pain. Pain evoked by either of these procedures was reported at 4 muscle groups, each assessed bilaterally: temporalis, masseter, lateral pterygoid area, and posterior mandibular and submandibular area; evoked pain was also reported at each temporomandibular joint (TMJ).

Jaw mobility in millimeters was measured under 6 conditions: pain-free opening; maximum unassisted opening; maximum assisted opening; left lateral excursion; right lateral excursion; and protrusion. In addition, pain evoked by any of these procedures was recorded.

TMJ noises: Examiners palpated the TMJs to detect joint noises (click, crepitus) during opening and during closing.

Palpation pain: Defined sites for each of the 10 muscle/joint groups were palpated by examiners' fingertips using $1 \mathrm{lb}$ (approximately $0.5 \mathrm{~kg}$ ) of force for the TMJs and intra-oral sites or $2 \mathrm{lb}$ (approximately $1 \mathrm{~kg}$ ) otherwise. Defined sites in three neck muscles were palpated bilaterally using $2 \mathrm{lbs}$ of pressure. Seven locations in the body were palpated bilaterally using $3 \mathrm{lbs}$ of pressure. For each palpation site, a positive or negative report of pain was recorded.

Tooth wear: Visual signs of facets representing at least $2 \mathrm{~mm}$ in length of surface wear at opposing tooth edges were recorded bilaterally from the incisor, cuspid, and pre-molar teeth. 


\section{Follow-up and Case-Classification of First-Onset TMD}

At three-monthly intervals after enrollment through May 2011, study participants were asked to complete a screening questionnaire (the Quarterly Health Update, QHU) that asked about TMD pain symptoms. Those reporting positive symptoms were invited to study clinics for a follow-up examination that determined presence or absence of painful TMD using the same clinical criteria as used in the initial examination. Specifically, the 260 incident cases satisfied two criteria for TMD: (1) symptoms of orofacial pain reported for 25 days in the prior 30 days; and (2) examiner findings of TMD myalgia, arthralgia, or both. Arthralgia was based on pain in temporomandibular joint(s) during jaw maneuver or digital palpation, and myalgia was based on pain during jaw maneuver or digital palpation in 23 of 8 muscle groups: temporalis, masseter, lateral pterygoid, and sub-mandibular and post-mandibular areas, each assessed bilaterally.

All examiners underwent annual training and calibration in the RDC/TMD methodology. In blinded, replicated examinations, Kappa statistics for inter-examiner reliability of TMD case-classification ranged from 0.82 to 1.0 , signifying excellent inter-examiner reliability between each of the study site examiners and OPPERA's reference examiner. Their reliability in classifying TMD case status is summarized elsewhere. ${ }^{2}$

\section{Statistical Analysis}

For descriptive purposes, the average annual incidence of first-onset TMD was calculated as the number of people with first-onset TMD divided by person-years of follow-up. To test hypotheses about associations between baseline risk factors and TMD incidence, univariate hazard ratios (HR) were computed using Cox proportional hazard regression. Hazard ratios represent the relative difference in hazard rates between two groups. While the hazard rate is a theoretical construct, representing the instantaneous probability of an event as the duration of follow-up approaches zero, it is a good approximation of the average incidence rate in a cohort study. Furthermore, the Cox proportional hazards models used to estimate hazard ratios require fewer statistical assumptions than other modeling methods. Hereafter, we use the term "incidence" when referring to the annual incidence rate and the hazard rate.

When the baseline risk factor was categorical (or binary), one category was nominated as the referent and dummy variables represented each of the other categories. For continuous variables (including count variables), scores were left in their natural metric. Hazard ratios were computed both with adjustment for study site and with additional adjustment for demographics: gender (male, female), age (years), race/ethnicity (white, African American/ Black, Hispanic, Asian, and other/unstated), and lifetime US residence (yes, and no/ unstated). Hazard ratios were also computed using multiple imputation to account for two sources of potential bias associated with: (a) non-examination of 243 people who developed symptoms as reported on the QHU but who did not return to the clinic within the required time-frame; and (b) a higher-than expected rate of TMD classification by one examiner who conducted 75 examinations. Further details can be found elsewhere. ${ }^{2}$

Two strategies of multivariable modeling were used to evaluate combined effects of baseline characteristics on rate of first-onset TMD. The first strategy used multivariable Cox regression models to evaluate contributions of major baseline variables. Because of the large number of clinical measures, exploratory factor analysis (EFA) was first undertaken using the 41 major univariate predictors (as listed in Tables 1-7). The approach utilized 4 steps widely used in exploratory item reduction methods: ${ }^{48}$ (1) variable selection and shaping; (2) evaluation of the correlation matrix; (3) factor extraction; and (4) rotation and interpretation. The EFA was conducted using the baseline data on a random selection of $50 \%$ of inception cohort; the remaining 50\% was reserved for confirmatory analyses not conducted at this 
time. During the EFA, some variables had response rates that were too conditional on other variables, and were dropped. Other variables, such as palpation scores of the masticatory muscles, exhibited expectedly high correlations among the different palpation variables resulting in collinearity, and so these variables were collapsed into a single measure. When only 1-2 predictor variables of a similar sort existed in the data set and consistently pointed to an underlying latent construct, they were eventually dropped from the EFA due to the occurrence of excessive factor loading and consequent negative residual variance. This is a common problem with factor models, wherein at least 3 predictors are necessary to create a robust factor. ${ }^{6}$

Each model was inspected for covariance coverage and variables with sparse covariance were dropped. The number of factors to retain was determined using the traditional criteria of eigenvalue > 1.0 and inspection of a scree plot; additionally, a parallel analysis was computed which determined the number of factors likely to emerge beyond chance alone. Parallel analysis estimates the number of factors to retain in a model by generating random data sets with the same number of observations and variables as in the original dataset. ${ }^{48}$ The eigenvalues are computed for each random dataset and averaged across datasets; when the average eigenvalue from the randomly constructed datasets is larger than the corresponding eigenvalue in the original dataset, then the factor associated with that eigenvalue is likely to be random noise. After extraction of factors, a geomin rotation was performed in order to enhance interpretation, and conventional fit statistics of Root Mean Square Error of Approximation (RMSEA) and Comparative Fit Index (CFI) were used to evaluate the adequacy of the factor model. ${ }^{6}$ Mplus ${ }^{29}$ was used for all EFA modeling; Mplus allows missing data, and final factor scores (z-scores) for the full sample were estimated by Mplus using the factor structure determined through exploration of the random 50\% of subjects.

Factor analysis produced 3 derived factor scores that were evaluated for associations with TMD incidence in a sequential set of multivariable models. Predictors in the first model were two pain-related factor scores along with study-site and socio-demographic variables as covariates. The second model added the jaw joint symptoms factor score along with three additional variables, each of which represented individual constructs not modeled by the EFA. Model 3 added single-item variables measuring trauma history and orthodontic treatment while Model 4 additionally included oral behaviors. The rationale for Model 1 was that it contained examination pain which is central to the case definition for TMD and any effects of jaw function of self-reported experiences should adjust for that pain. Likewise, oral parafunction was entered as the last block, in part because of the suspected role as both cause and consequence of pain, to assess its independent contribution to TMD incidence. Given this a priori plan for exploratory analysis of multivariable associations, interactions were not tested.

The second strategy used random forest modeling 22 to analyze potential contributions of all variables, not merely the reduced set used in the multivariable Cox model. The random forest model was created by generating a set of decision trees. A decision tree predicts an outcome by recursively partitioning the set of predictor variables producing results that can be visualized as a tree diagram. ${ }^{4}$ The number of predictors in each tree was chosen to be the square root of the number of observations, which is a conventional approach used in random forest modeling.

This novel method of data mining was used to achieve two goals: a) to identify the most important risk factors for first-onset TMD; and b) to generate plots depicting adjusted association between each variable and TMD incidence, with adjustment for the effects of other variables and with latitude in generating the plots that permitted departure from a 
straight-line association. The model produced importance scores, one for each variable, representing the decrease in the predictive accuracy of the model when the variable is measured incorrectly. The most important variable was assigned a score of 100, and all other importance scores have lower values that could range to a negative value if the variable worsened prediction. The random forest model was used also to compute the expected rate of first-onset TMD that would be observed at several values of the variable after averaging over the values of all other variables in the model. Partial dependence plots were then generated and LOESS smoothing was used to help visualize the association. ${ }^{23}$

The two strategies were selected in favor of other approaches for multivariable analysis for several reasons. The first strategy is a conventional approach that adjusts for potential confounding effects of variables identified a priori, based on conceptual relevance and univariate association with TMD incidence. However, it does not take advantage of information about the excluded variables. Thus, a random forest model was used to evaluate contributions of all variables. Random forests have several other advantages compared to conventional linear regression models. Specifically, random forests can impute for missing data and handle large numbers of correlated predictor variables without decreasing the accuracy of the model. ${ }^{20}$

\section{RESULTS}

The cohort of 2,737 initially TMD-free people was followed for a total of 7,404 personyears (median $=2.8$ years/person), during which time 260 people developed first-onset TMD, yielding an annual incidence rate of $3.5 \%$ per annum.

\section{Univariate results}

For most variables, hazard ratios (HR) and 95\% confidence limits (CL) were generally consistent in unimputed models of observed data that either adjusted for study site or study site and socio-demographics. Most HRs did not change appreciably in the analysis that also imputed outcomes for people who were not examined as intended. A few HRs, however, were less stable. In the text that follows, emphasis is given to the HRs from imputation given that two sources of potential bias were corrected in the computation of the HR estimates.

Putative etiologic factors (Table 1)—Of the three types of trauma queried, only injury due to prolonged opening was predictive of first-onset TMD although HRs varied across models and was not significantly different from 1.0 in the imputed estimate. A prior history of orthodontics did not predict TMD incidence (HR=0.85). Parafunctional oral behavior summary scores in the highest tercile predicted TMD incidence, HR=1.75 (CL 1.28, 2.39) relative to the lowest tercile.

Clinical status by self-report-The 119 TMD-free people at baseline who reported $\geq 3$ non-specific orofacial symptoms had more than twice the incidence of TMD as people with $<3$ such symptoms (Table 2). Facial pain in the 6-months before baseline was reported by a small number of participants $(n=272)$ and even low intensity pain of GCPS Grade I was associated with greater incidence of TMD. An increase of 1 SD in characteristic pain intensity at baseline was associated with a modest, though statistically significant increase in TMD incidence (HR=1.18, CL 1.08, 1.27, Table 3). Likewise, a 1 SD increase in the number of days with at least $50 \%$ decreased efficiency due to orofacial pain was associated with significantly greater TMD incidence. Scores from the JFLS were not significantly associated with TMD incidence. All of the variables in Table 3 were highly skewed as indicated by a minimum value of zero for the 1 st and 2 nd terciles of all variables, indicating that only a 
small number of participants reported pain, interference, or functional limitation at baseline enrollment.

Self-reported TMJ noises at baseline were reported by more than 500 people and were associated with significant increases in TMD incidence (Table 4). Inability to open the jaw widely was also a significant predictor of TMD incidence, although it was reported less frequently than TMJ noises. In contrast, inability to close the jaw from a wide-open position was not a significant predictor of TMD incidence.

Clinical status by examination-None of the examiner-assessed measures of jaw mobility was a significant predictor of TMD incidence (Table 5). In contrast, people with pain during jaw opening had approximately 50\% greater incidence of TMD (i.e., statistically significant HRs of approximately 1.5) compared to people who reported no pain during such procedures (Table 6). Self-reported history of TMJ noises predicted TMD incidence; in contrast, examiner-assessment of joint noises did not significantly predict TMD incidence (Table 6).

Pain during palpation of the masticatory muscles and TM joints was a significant predictor of TMD, with HRs ranging from 1.4 to 1.6 according to the muscle-group assessed (Table 7). The HRs are presented for each muscle, by left and right side, in order to demonstrate the interesting pattern of anatomical symmetry. Compared to the temporalis and masseter muscles, HRs were less for the posterior and sub-mandibular area and some ceased to be significant in the imputation models. In addition, greater numbers of palpation tenderpoints in both the neck and the body were associated with greater incidence of TMD (Table 5).

\section{Factor analysis}

Models containing 3, 4, and 5 factors were entertained, and all yielded latent constructs that were readily interpretable. However, models based on 4 or 5 factors were the result of overextraction and contained negative residuals. The scree and parallel analysis jointly pointed to the selection of a 3 -factor model (Figure 1), which accounted for $72 \%$ of the variance and is an acceptable outcome. The final model was built from 25 observed variables; additional variable reduction had occurred during model fitting in the form of collapsing variables in order to eliminate collinearity effects, such that 15 variables contributed to the final model.

The observed factors in the final, 3-factor model were labeled Jaw Mobility Pain (from both opening and lateral and protrusive movements of the jaw), Palpation Pain (from pain responses to palpation of the muscles of mastication, each TMJ, neck, and body), and TMJ Function by History (TMJ clicking, click-related pain, and locking prior to the baseline clinic visit) (Table 8). The first two factors scale in the direction of pathology, while the third factor scales in the direction of health due to the coding of the source variables. The RMSEA was 0.062 (90\% CL 0.056, 0.067), which represents slightly more misfit than the traditionally desired threshold of 0.050 for the RMSEA. This degree of misfit seemed justifiable because the variables fitted within a plausible model with stable factor loadings. ${ }^{6}$ The CFI was 0.980 , indicating excellent fit. The factor correlations of this intentionally oblique final model were 0.34 (F1 \& F2), -0.07 (F1\& F3), and -0.17 (F2 \& F3).

The factor structure was applied to the full data set in order to compute factor scores. The factor score correlations were 0.57 (F1 \& F2), -0.25 (F1\& F3), and -0.21 (F2 \& F3) which justified the retention of the 3 factors for subsequent modeling. After the factor scores were extracted from the full sample, scores for the third factor were reflected in order to scale in the direction of pathology. The three factors were then used in the subsequent multivariate analyses. 


\section{Multivariable relationships}

Both pain-related factor scores were significantly associated with TMD incidence in the demographically-adjusted Model 1. The factor measuring pain from vertical jaw opening continued to be a significant predictor in all subsequent models, while the factor measuring pain from palpation was attenuated slightly, and in so doing, it became statistically nonsignificant in Models 2, 3 and 4. The attenuation apparently was due to the effects of face/ jaw symptoms, which was a significant predictor in Model 2. HRs from Model 2 remained virtually unchanged after additional adjustment for history of injury and orthodontic treatment (Model 3). In the fully-adjusted Model 4, both pain from vertical jaw mobility and face/jaw symptoms remained statistically significant predictors, and two other variables made smaller contributions: pain from palpation $(\mathrm{HR}=1.14, \mathrm{P}=0.096)$ and oral parafunctional behaviors $(\mathrm{HR}=1.14, \mathrm{P}=0.052)$.

\section{Random forest plots}

The second multivariable approach of random forest models found that parafunctional oral behaviors (OBC sum score) had the most pronounced effect on TMD from among the individual clinical measures; it was therefore assigned a variable importance score (VIS) of 100. The second and third most important predictors were inability to open the mouth wide for any reason in the last month (VIS=34.5) and number of non-specific orofacial symptoms (VIS=26.5). The next two most important predictors were pain from palpation of right masseter (VIS=9.2) and right temporalis (VIS=8.4). The fall-off in the VIS is notable, such that only the first three variables listed had substantial independent relationships with TMD incidence.

Partial dependence plots of these three strongest predictors were selected from the random forest model in order to illustrate their effects (see Figure 2). For the OBC sum score (Figure $2 \mathrm{~A}$ ), the predicted TMD incidence increased linearly from a threshold score of about 20 although there were substantial outliers from the LOESS curve above OBC scores of 40. People who reported inability to open the mouth wide for any reason in the month prior to enrolment had markedly greater TMD incidence than people who did not (Figure 2B). Although not plotted, a similar relationship was seen for the same symptom experienced prior to one month before baseline. The number of non-specific orofacial symptoms (Figure 2C) exhibited a relatively linear relationship across the number of symptoms.

\section{DISCUSSION}

During the median 2.8 year follow-up period among initially TMD-free adults, greater TMD incidence was predicted by several self-reported orofacial characteristics recorded at baseline while examiner-assessed characteristics were less important. In univariate analysis, self-reported oral parafunctional behaviors and both orofacial pain symptoms and nonspecific (non-pain) orofacial symptoms were significant predictors of TMD incidence. However, reported jaw function was not. Examiner-evoked pain from jaw opening or muscle and TMJ palpation was a significant univariate predictor of TMD incidence, but it was of marginal significance in multivariable models that adjusted for self-reported characteristics. Importantly, all of these significant predictors represent "pre-clinical" characteristics, in that they were evaluated at baseline, in people who had not yet developed TMD. Some of these predictors (e.g., parafunction) represent characteristics associated with TMD. Others (e.g., pain from jaw opening) represent characteristics used as diagnostic criteria, although in this study their levels were below the threshold needed for TMD caseclassification. While selected individual variables at enrollment may have denoted a preclinical state, with regard to TMD, the inter-relationship among them, especially in light of the case definition, is revealing. At baseline, individuals who subsequently developed first 
onset TMD reported pain with unassisted or assisted jaw opening yet their opening range did not differ from those who did not develop TMD; those same individuals with pain on jaw opening reported, via the JFLS, an absence of any limitation in opening and an absence of any limitation in chewing. Therefore, individuals who did report pain with opening at baseline were without measurable limitations in either opening or function. Those negative characteristics (e.g., absence of recent pain by history, absence of limitation in jaw functions) would be inconsistent with the presence of diagnosable musculoskeletal pain at baseline.

Meanwhile, several hypothesized risk factors were not significant predictors, even in univariate analysis, including self-reported history of external trauma and examiner-assessed TMJ noises, tooth wear and range of jaw motion. The pattern of these findings, taken together, was more consistent with the central dysregulation model described previously.

Oral parafunction (e.g., clenching the teeth during the day, or holding the jaw rigid) was the strongest predictor of TMD incidence in the multivariate random forest model. In the multivariate Cox regression model, parafunction was at the threshold of statistical significance, even after adjustment for orofacial symptoms and examination findings. The relevance of oral parafunction in the etiology of TMD has been speculated for over 50 years but only a few longitudinal studies have attempted to clarify its contribution. Among university students, multiple oral parafunctional behaviors, compared to trauma, had a threefold stronger effect in predicting TMD symptoms 3 years later. ${ }^{1}$ Among individuals who sought treatment for TMD, sleep bruxism reported when enrolled by late adolescents predicted subsequent treatment-seeking 10 years and again 20 years after enrollment; at each follow-up assessment, oral parafunction was an increasingly strong characteristic. ${ }^{7}$ A range of cross-sectional studies of primarily chronic TMD (e.g., $8,16,28,30,41,49,53$ ) consistently demonstrates a strong relationship with parafunctional behaviors; the strength of parafunction as a predictor of first-onset TMD suggests that the strong relationship of parafunction in chronic TMD, as previously reported from the case-control OPPERA study, ${ }^{32}$ may well originate in behaviors operating prior to symptom onset.

In the present study, where the validated ${ }^{25,31,36} \mathrm{OBC}$ measured parafunctional behaviors, people in the upper tercile of the scale exhibited elevated TMD incidence. The upper tercile threshold of $\geq 25$ (range: 0-84) would be reached by a person who, for example, reported performing 17 of the behaviors "a little of the time" and the four other behaviors "some of the time". Likewise, the threshold would be reached by a person who reported performing 7 functions "all the time". In other words, the people at increased risk reported parafunctional behaviors that were pervasive, either in the large number of type of behaviors or the very high frequency of a small number of types of behaviors. The sheer density of parafunctional behaviors required to increase risk suggests underlying central dysregulation in the form of overactive motor activation, underactive motor inhibition, loss of normal proprioception, and/or persistent psychophysiologic reactivity. The hypothesized central dysregulation may be specific to the masticatory system, as measured here, or may include more general motor activation in addition to that specific to the masticatory system. In either case, mediation via CNS dysregulation is an obvious further research area, ${ }^{10,26,50}$ as is possible overuseinduced peripheral myofascial tissue changes. ${ }^{9}, 17,27,42$ Future OPPERA analyses will investigate the particular behaviors associated with incidence as well as the possibility of CNS dysregulation using genetic data and other phenotypes to evaluate their associations with parafunction.

It was noteworthy that TMD incidence was so strongly predicted by a count of 6 nonspecific orofacial symptoms described in terms other than "pain". The association persisted in both multivariable models, even after adjustment for painful symptoms and examination 
findings. The 6 questionnaire items, which included ache, pressure, and fatigue, were asked in order to capture experiences that were presumably aversive but did not represent the experience of "pain" used for TMD case-classification. At enrollment, one sixth of people reported at least one non-specific orofacial symptom, and in the random forest model, there was a distinct, monotonic association between greater numbers of such symptoms and TMD incidence. One explanation for the association is that these non-pain words represent preclinical TMD and therefore the subsequent conversion to acute TMD was not surprising. However, the association between the non-pain words and subsequent TMD onset remains even after adjusting for pain in the model, suggesting that the neurobiology underlying the semantics of these words is probably different from "pain". Another possibility is that these non-pain words reflect dysphoric body experiences that may serve as a proxy for "somatic symptoms", which was the strongest psychosocial predictor of TMD incidence in this same cohort. ${ }^{15}$ However, based on data reported elsewhere in this volume, that also seems unlikely. ${ }^{3}$

Symptoms provoked specifically by jaw movement were also strong predictors of TMD incidence, both in univariate and multivariate analysis. In the random forest model, restricted opening for any reason emerged as the most important jaw-movement-related symptom, whereas in the multivariate Cox model, pain evoked during jaw motion in the examination (i.e., "jaw mobility pain" derived from factor analysis) emerged. The fact that one multivariate method chose "restriction for any reason" while the other chose "jaw mobility pain" is probably related more to methodology than to substantive differences in types of jaw movement. Specifically, psychometric principles propose that greater measurement fidelity will usually be achieved by a reliable factor score than by its individual components. This greater fidelity probably contributed to the selection of the "jaw mobility pain" score in the Cox model. In contrast, the random forest model was purposefully constructed with component items, not factor scores. This nuance illustrates one advantage of factor analytic methods in data reduction; on the other hand, the random forest model points to a single item—self-reported assessment of jaw motion—as a powerful predictor of TMD incidence and serves as a check on possible loss of important detail when subsuming individual variables within a factor.

The absence of meaningful associations between history of external jaw trauma and TMD incidence is in contrast to cross-sectional studies (e.g., ${ }^{5,21}$ ), where TMD cases are much more likely than controls to report such a history. For example, in the OPPERA baseline case-control study, odds ratios ranged from 4.2 to 8.3 for the same questions about trauma that, in this analysis, were barely associated with TMD incidence at the univariate level, and not at all in the multivariate models. ${ }^{32} \mathrm{~A}$ possible interpretation is attribution bias. However, it is also possible that trauma contributes to TMD only in the immediate aftermath of injury or contributes to TMD via a delayed response through subsequent physiological events (e.g., sensitization ${ }^{5}$ ) that then promote the transition from acute to chronic TMD. In this paper, traumatic episodes after enrollment were not analyzed; data collected at three monthly intervals during follow-up are now being analyzed.

Other characteristics cited widely as likely etiological influences were not significant predictors of TMD. They included clinically-assessed extent of jaw opening and tooth wear. Neither was TMD onset predicted by self-reported jaw functional limitation. In contrast, jaw functional limitation was strongly associated with chronic TMD in the OPPERA baseline case-control study. A likely explanation is that important levels of functional limitation occur after TMD develops, whereas the severely truncated levels of functional limitation reported at baseline in this cohort were insufficient by themselves to predict risk of developing TMD. 
One limitation of this study was its restriction to risk factors measured at baseline. TMD often develops slowly, with pre-clinical phases that often resolve. ${ }^{43}$ It is probable that distinct elements of TMD pathophysiology evolve over time to be diagnosed clinically as TMD only when multiple etiological influences coincide creating a sufficient-componentcause. ${ }^{38}$. The accompanying papers in this volume $2,3,15,19,39,44$ clearly highlight that no single risk factor or environmental exposure for developing TMD has yet been identified as a necessary or sufficient cause (using the language of classical causation). However, with respect to complex disease, the necessary and sufficient concepts of classical causation may not be relevant. To determine how risk factors unfold over time, they need to be measured and analyzed at repeated intervals prior to onset of TMD. Some other putative risk factors have been measured repeatedly in this study and will be reported in future analysis. This limitation should not distract from the equally important finding that distant events of external trauma, yawn trauma, and open-locking of the jaw did not contribute to TMD incidence; this is often a question that emerges in TMD patient histories.

A second limitation was that no imaging procedures were used to characterize TMJ status. Radiologic evidence of disc derangement therefore was not evaluated, either as a baseline predictor of TMD or as an outcome in its own right. While imaging methods such as Magnetic Resonance (MR) are highly informative as to disc status, the method is not feasible in a large-scale, population-based study such as OPPERA. Moreover, MRdetermined changes in disc status correspond poorly to pain complaints. ${ }^{37,46}$. Consequently, we do not regard this limitation as confounding the present results. Finally, the therapeutic importance of MR-determined disc position is not clear, in that the clinical presentation of a painful clicking joint requires clinical decision-making with respect to possible intervention, regardless of whether imaging-based classification indicates a disc as displaced or not. ${ }^{34}$

In summary, in this cohort some orofacial examination findings contributed to TMD incidence, although the magnitude of the effects was small, and some long-held clinical signs did not predict incidence at all. More pronounced influences were observed for symptoms, particularly those that seemed to reflect dysregulation in systems beyond the masticatory tissues. For example, extensive oral parafunction and non-specific symptoms, both suggestive of systemic dysregulation, were strong predictors of TMD incidence, whereas symptoms consistent with local change in masticatory tissues (e.g., joint noises determined by examination) were not. This interpretation is consistent with the OPPERA heuristic ${ }^{24}$ by suggesting a prominent contribution of systems beyond the masticatory tissues to the incidence of first-onset TMD. These findings help refine our understanding of what TMD means as a regional disorder.

\section{Acknowledgments}

The authors would like to thank the OPPERA Research Staff for their invaluable contributions to this work. In addition, we express our gratitude to the research participants who have devoted time and effort in support of this research.

This work was supported by NIH grant U01DE017018 and P01NS045685. The OPPERA program also acknowledges resources specifically provided for this project by the respective host universities: University at Buffalo, University of Florida, University of Maryland-Baltimore, and University of North Carolina-Chapel Hill. Roger Fillingim and Gary Slade are consultants and equity stock holders, and William Maixner and Dr. Luda Diatchenko are cofounders and equity stock holders in Algynomics, Inc., a company providing research services in personalized pain medication and diagnostics. 


\section{REFERENCES}

1. Akhter R, Morita M, Esaki M, Nakamura K, Kanehira T. Development of temporomandibular disorder symptoms: a 3-year cohort study of university students. J Oral Rehabil. 2011; 38:395-403. [PubMed: 21294762]

2. Bair E, Brownstein NC, Ohrbach R, Greenspan JD, Dubner R, Fillingim RB, Diatchenko L, Smith S, Maixner W, Gonzalez Y, Gordon S, Lim P-F, Ribeiro-Dasilva M, Dampier D, Knott C, Slade GD. Study design, methods, sample characteristics and loss-to-follow-up: the OPPERA prospective cohort study. Journal of Pain. 2013 In press.

3. Bair E, Ohrbach R, Fillingim RB, Greenspan JD, Dubner R, Diatchenko L, Knott C, Maixner W, Slade GD. Multivariable modeling of phenotypic risk factors for first-onset TMD: the OPPERA prospective cohort study. Journal of Pain. 2013 In press.

4. Breiman, L.; Friedman, J.; Olshen, R.; Stone, C. Classification and Regression Trees. Wadsworth; New York: 1984.

5. Burgess J. Symptom characteristics in TMD patients reporting blunt trauma and/or whiplash injury. J Craniomandib Disord Facial Oral Pain. 1991; 5:251-7.

6. Byrne, BM. Structural Equation Modeling with Mplus: Basic Concepts, Applications, and Programming. Routledge Academic; 2011.

7. Carlsson GE, Magnusson T, Egermark I. Prediction of demand for treatment of temporomandibular disorders based on a 20-year follow-up study. J Oral Rehabil. 2004; 31:511-7. [PubMed: 15189306]

8. Celic R, Jerolimov V, Panduric J. A study of the influence of occlusal factors and parafunctional habits on the prevalence of signs and symptoms of TMD. International Journal of Prosthodontics. 2002; 15:43-8. [PubMed: 11887598]

9. Chung JW, Ohrbach R, McCall WD Jr. Characteristics of electrical activity from myofascial trigger points. Clinical Neurophysiology. 2006; 117:2459-66. [PubMed: 16949863]

10. Chung JW, Ohrbach R, McCall WD Jr. Effect of Increased Sympathetic Activity on Electrical Activity from Myofascial Painful Areas. American Journal of Physical Medicine and Rehabilitation. 2004; 83:842-50. [PubMed: 15502737]

11. Clark GT. Etiologic theory and the prevention of temporomandibular disorders. Advances in Dental Research. 1991; 5:60-6. [PubMed: 1819285]

12. Clark, GT.; Solberg, WK.; Monteiro, AA. Temporomandibular Disorders: New Challenges in Clinical Management, Research, and Teaching.. In: Clark, GT.; Solberg, WK., editors. Perspectives in Temporomandibular Disorders. Quintessence; Chicago: 1987. p. 13-26.

13. de Boever JA. Functional disturbances of temporomandibular joints. Oral Sciences Reviews. 1973; 2:100-17. [PubMed: 4515361]

14. Dworkin SF, LeResche L. Research Diagnostic Criteria for Temporomandibular Disorders: Review, Criteria, Examinations and Specifications, Critique. J Craniomandib Disord Facial Oral Pain. 1992; 6:301-55.

15. Fillingim RB, Ohrbach R, Greenspan JD, Knott C, Diatchenko L, Dubner R, Bair E, Baraian C, Mack N, Slade GD, Maixner W. Psychosocial factors associated with development of TMD: the OPPERA prospective cohort study. Journal of Pain. 2013 In press.

16. Gavish A, Halachmi M, Winocur E, Gazit E. Oral habits and their association with signs and symptoms of temporomandibular disorders in adolescent girls. J Oral Rehabil. 2000; 27:22-32. [PubMed: 10632840]

17. Gerwin RD. A review of myofascial pain and fibromyalgia--factors that promote their persistence. Acupuncture in Medicine. 2005; 23:121-34. [PubMed: 16259310]

18. Greene CS, Mohl ND, McNeill C, Clark GT, Truelove EL. Temporomandibular disorders and science: a response to the critics. J Prosthet Dent. 1998; 80:214-5. [PubMed: 9710825]

19. Greenspan JD, Slade GD, Bair E, Dubner R, Fillingim RB, Ohrbach R, Knott C, Diatchenko L, Liu $\mathrm{Q}$, Maixner W. Pain sensitivity and autonomic factors associated wtih development of TMD: the OPPERA prospective cohort study. Journal of Pain. 2013 In press.

20. Hastie, T.; Tibshirani, R.; Friedman, J. The elements of statistical learning: data mining, inference, and prediction. Second ed.. Springer; New York: 2009. 
21. Huang GJ, LeResche L, Critchlow CW, Martin MD, Drangsholt MT. Risk factors for diagnostic subgroups of painful temporomandibular disorders (TMD). J Dent Res. 2002; 81:284-8. [PubMed: 12097315]

22. Ishwaran H, Kogalur UB, Blackstone EH, Lauer MS. Random survival forests. Annals of Applied Statistics. 2008; 2:841-60.

23. Loader, C. Local Regression and Likelihood. Springer; New York: 1999.

24. Maixner W, Diatchenko L, Dubner R, Fillingim RB, Greenspan JD, Knott C, Ohrbach R, Weir B, Slade GD. Orofacial Pain Prospective Evaluation and Risk Assessment Study - The OPPERA Study. Journal of Pain. 2011; 12:T4-T11. [PubMed: 22074751]

25. Markiewicz MR, Ohrbach R, McCall WD Jr. Oral Behaviors Checklist: Reliability of Performance in Targeted Waking-state Behaviors. J Orofacial Pain. 2006; 20:306-16. [PubMed: 17190029]

26. Melzack R. Phantom limbs, the self and the brain. Can Psychol. 1989; 30:1-16.

27. Mense S. Nociception from skeletal muscle in relation to clinical muscle pain. Pain. 1993; 54:24191. [PubMed: 8233542]

28. Miyake R, Ohkubo R, Takehara J, Morita M. Oral parafunctions and association with symptoms of temporomandibular disorders in Japanese university students. J Oral Rehabil. 2004; 31:518-23. [PubMed: 15189307]

29. Mplus User's Guide [computer program]. Version 3. Muthén \& Muthén; Los Angeles: 2004.

30. Nilner M. Relationships between oral parafunctions and functional disturbances in the stomatognathic system among 15- to 18-year-olds. Acta Odontol Scand. 1983; 198:197-201. [PubMed: 6578661]

31. Ohrbach R. Assessment and further development of RDC/TMD Axis II biobehavioural instruments: a research programme progress report. J Oral Rehabil. 2010; 37:784-98. [PubMed: 20701668]

32. Ohrbach R, Fillingim RB, Mulkey F, Gonzalez Y, Gordon S, Gremillion H, Lim PF, RibeiroDasilva M, Greenspan JD, Knott C, Maixner W, Slade G. Clinical findings and pain symptoms as potential risk factors for chronic TMD: Descriptive data and empirically identified domains from the OPPERA case-control study. Journal of Pain. 2011; 12:T27-T45. [PubMed: 22074750]

33. Ohrbach R, Granger CV, List T, Dworkin SF. Pain-related Functional Limitation of the Jaw: Preliminary Development and Validation of the Jaw Functional Limitation Scale. Comm Dent Oral Epidem. 2008; 36:228-36.

34. Ohrbach R, Greene C. Temporomandibular joint diagnosis: striking a balance between the sufficiency of clinical assessment and the need for imaging. Oral Surgery Oral Medicine Oral Pathology Oral Radiology. 2013; 116:124-5.

35. Ohrbach R, Larsson P, List T. The Jaw Functional Limitation Scale: Development, reliability, and validity of 8-item and 20-item versions. J Orofacial Pain. 2008; 22:219-30. [PubMed: 18780535]

36. Ohrbach R, Markiewicz MR, McCall WD Jr. Waking-state oral parafunctional behaviors: specificity and validity as assessed by electromyography. European Journal of Oral Sciences. 2008; 116:438-44. [PubMed: 18821986]

37. Ribeiro RF, Tallents RH, Katzberg RW, Murphy WC, Moss ME, Magalhaes AC, Tavano O. The prevalence of disc displacement in symptomatic and asymptomatic volunteers aged 6 to 25 years. J Orofacial Pain. 1997; 11:37-47. [PubMed: 10332309]

38. Rothman, KJ.; Greenland, S.; Lash, TL. Modern Epidemiology. 3rd ed.. Wolters Kluwer Health/ Lippincott Williams \& Wilkins; Philadelphia: 2008.

39. Sanders AE, Slade GD, Bair E, Fillingim RB, Knott C, Dubner R, Greenspan JD, Maixner W, Ohrbach R. General health status and incidence of first-onset temporomandibular disorder: OPPERA prospective cohort study. Journal of Pain. 2013 In press.

40. Schiffman E, Ohrbach R, Truelove E, Look J, Anderson G, Goulet J-P, et al. Diagnostic Criteria for Temporomandibular Disorders (DC/TMD) for Clinical and Research Applications: Recommendations of the International RDC/TMD Consortium Network and Orofacial Pain Special Interest Group. In review. 2013

41. Schiffman EL, Fricton JR, Haley D. The relationship of occlusion, parafunctional habits and recent life events to mandibular dysfunction in a non-patient population. J Oral Rehabil. 1992; 19:20123. [PubMed: 1500964] 
42. Shah JP, Phillips TM, Danoff JV, Gerber LH. An in vivo microanalytical technique for measuring the local biochemical milieu of human skeletal muscle. J Appl Physiol. 2005; 99:1977-84. [PubMed: 16037403]

43. Slade G, Sanders A, Bair E, Brownstein NC, Dampier D, Knott C, Fillingim R, Maixner WO, Smith S, Greenspan J, Dubner R, Ohrbach R. Preclinical episodes of orofacial pain symptoms and their association with healthcare behaviors in the OPPERA prospective cohort study. Pain. 2013; 154:750-60. [PubMed: 23531476]

44. Slade GD, Bair E, Greenspan JD, Dubner R, Fillingim RB, Diatchenko L, Maixner W, Knott C, Ohrbach R. Signs and symptoms of first-onset TMD and socio-demographic predictors of its development: the OPPERA prospective cohort study. Journal of Pain. 2013 In press.

45. Tallents RH. Etiologic theory and prevention of temporomandibular joint disorders: Reaction paper. Advances in Dental Research. 1991; 5:67-8. [PubMed: 1819286]

46. Tallents RH, Katzberg RW, Murphy W, Proskin H. Magnetic resonance imaging findings in asymptomatic volunteers and symptomatic patients with temporomandibular disorders. The Journal of Prosthetic Dentistry. 1996; 75:529-34. [PubMed: 8709019]

47. Tallents, RH.; Stein, SI.; Moss, ME. The role of occlusion in temporomandibular disorders.. In: Fonseca, RJ., editor. Oral and Maxillofacial Surgery: Temporomandibular Disorders. Vol. 4. W.B. Saunders; Philadelphia: 2000. p. 194-237.

48. Thompson B, Daniel LG. Factor analytic evidence for the construct validity of scores: A historical overview and some guidelines. Educational and Psychological Measurement. 1996; 56:197-208.

49. van der Meulen MJ, Lobbezoo F, Aartman IHA, Naeije M. Self-reported oral parafunctions and pain intensity in temporomandibular disorder patients. J Orofacial Pain. 2006; 20:31-5. [PubMed: 16483018]

50. van Selms MK, Lobbezoo F, Wicks DJ, Hamburger HL, Naeije M. Craniomandibular pain, oral parafunctions, and psychological stress in a longitudinal case study. J Oral Rehabil. 2004; 31:73845. [PubMed: 15265208]

51. Von Korff, M. Epidemiologic and survey methods: chronic pain assessment.. In: Turk, DC.; Melzack, R., editors. Handbook of Pain Assessment. Guilford Press; New York: 1992. p. 389-406.

52. Von Korff M, Ormel J, Keefe FJ, Dworkin SF. Grading the severity of chronic pain. Pain. 1992; 50:133-49. [PubMed: 1408309]

53. Winocur E, Gavish A, Finkelshtein T, Halachmi M, Gazit E. Oral habits among adolescent girls and their association with symptoms of temporomandibular disorders. J Oral Rehabil. 2001; 28:624-9. [PubMed: 11422693] 


\section{PERSPECTIVE}

OPPERA's prospective cohort study identifies predictors of first-onset TMD comprising self-reported orofacial symptoms and examination findings. The results suggest a complex pattern of TMD etiology that is influenced by disorders locally, in masticatory tissues, and systemically, in pain-regulatory systems. 


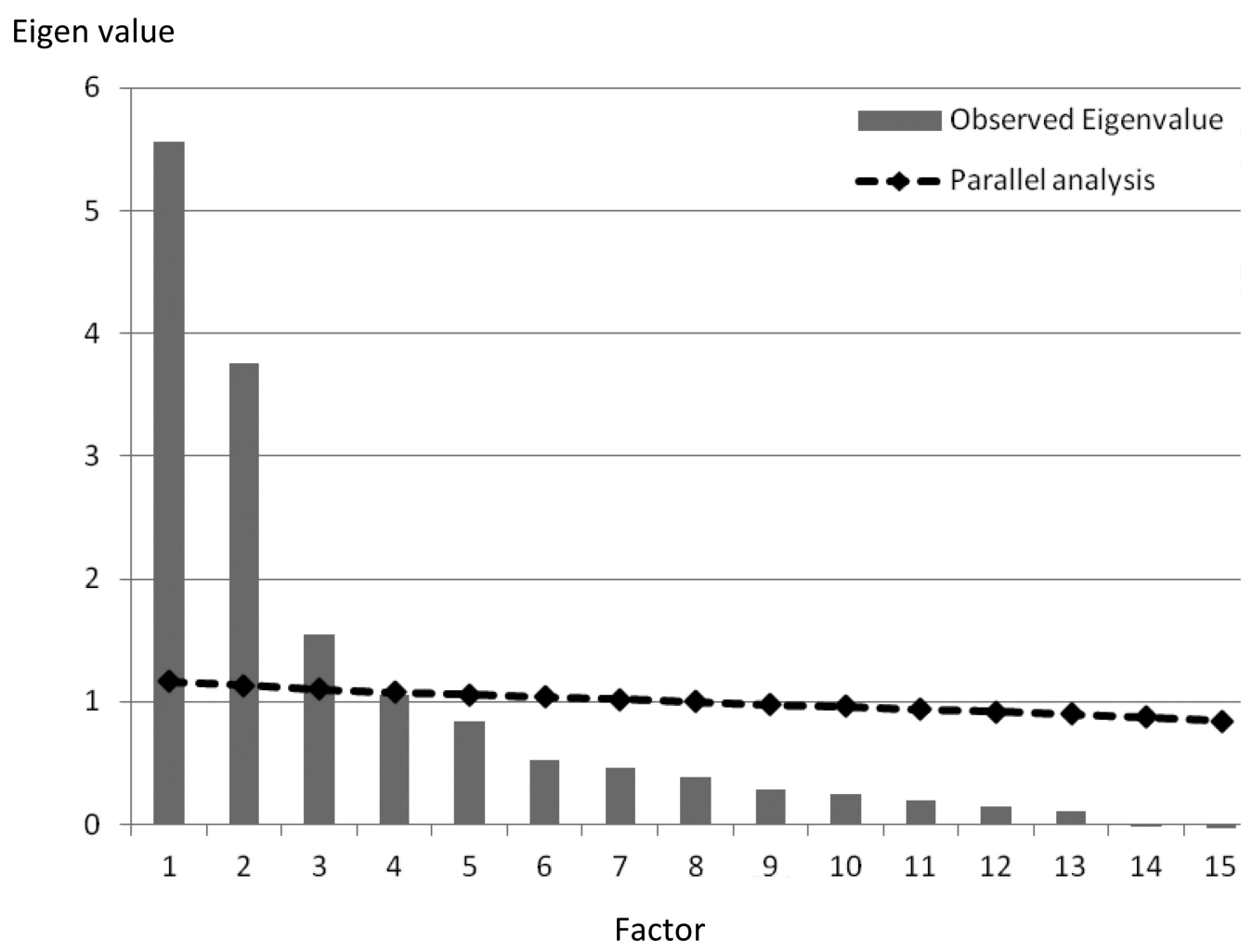

Figure 1. Scree plot and parallel analysis from exploratory factor analysis (EFA)

The vertical lines depict the eigenvalues for each factor, showing a turn at factor 3 . The solid line depicts the upper bound of the $95 \%$ confidence interval for eigenvalues that would be expected for each factor from an EFA computed on a random dataset of the same size as the actual data. As can be seen, the observed eigenvalues for the first 3 factors exceed the chance line, consistent with the scree plot interpretation. 


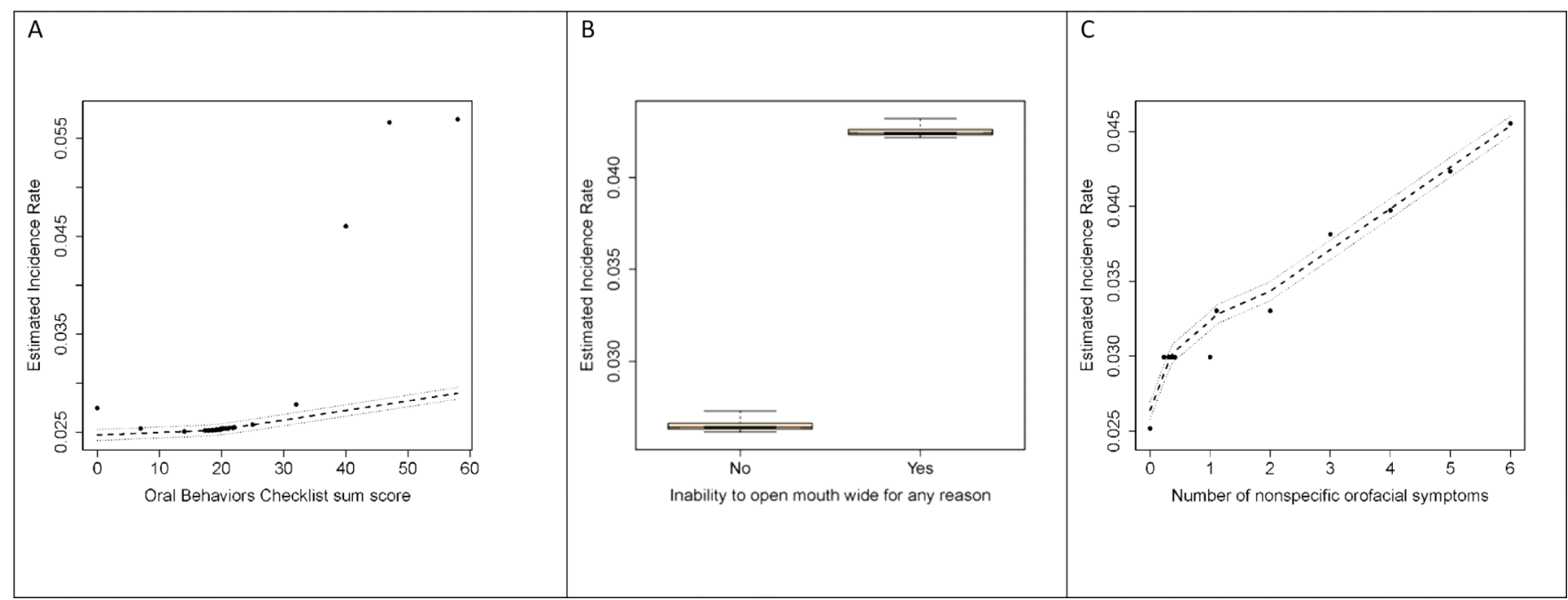

Figure 2. Predicted TMD incidence rates from random forest models: OPPERA prospective cohort study, 2006-2011

TMD incidence rates, expressed as cases per 100-person-years, were generated from random forest models that predicted TMD onset using study site and clinical orofacial characteristics reported in Tables 1-7. Predicted values (O) are plotted together with LOESS-smoothed estimates (- - ) and their $95 \%$ confidence intervals (…). A: Sum of the Oral Behaviors Checklist (OBC) is the simple sum of an ordinal response scale for each of 21 items. B: Inability to open mouth was reported as yes/no. C: Number of face jaw symptoms is the simple count of 'yes' responses to each of 6 non-specific orofacial symptoms. 


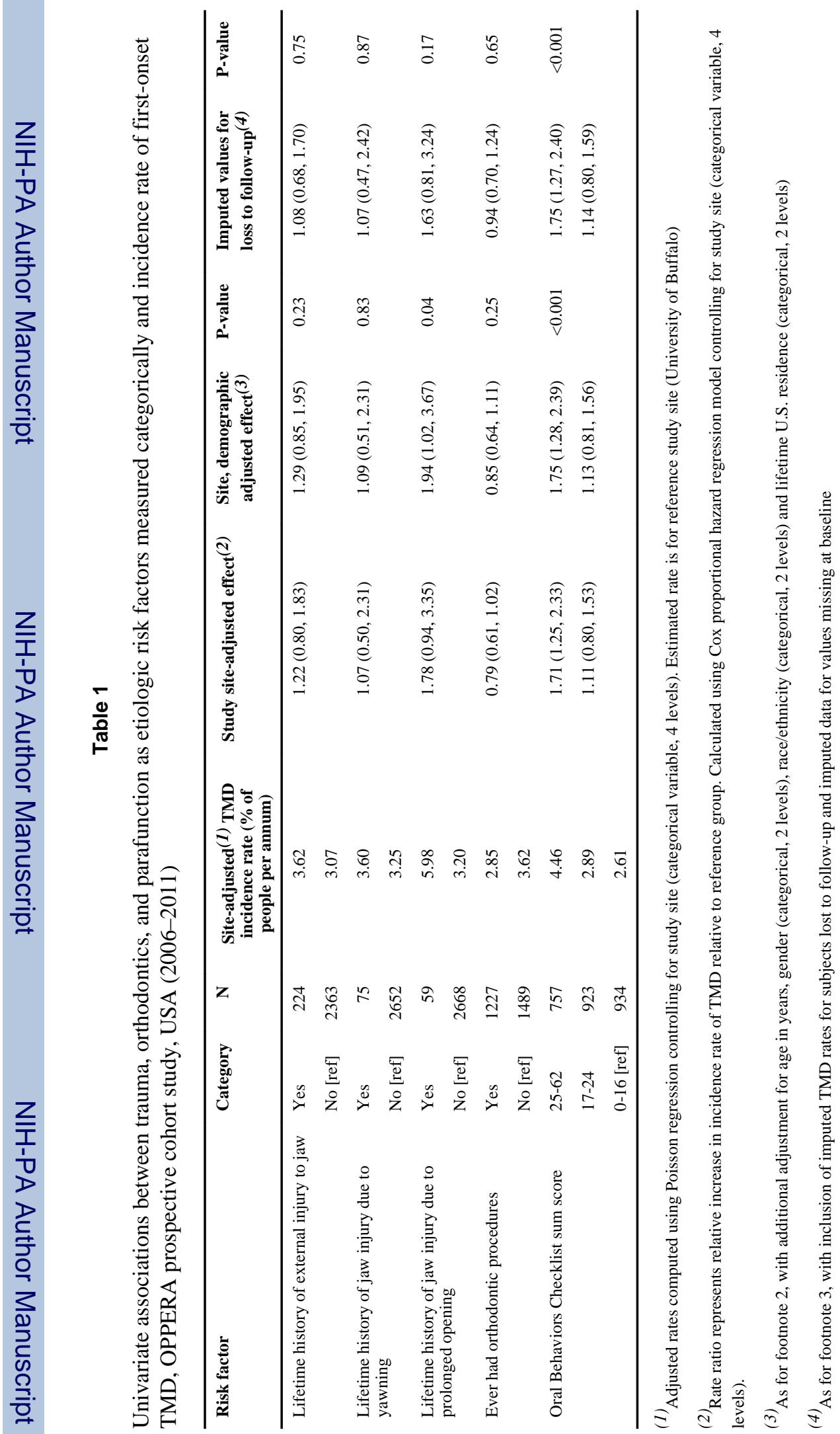




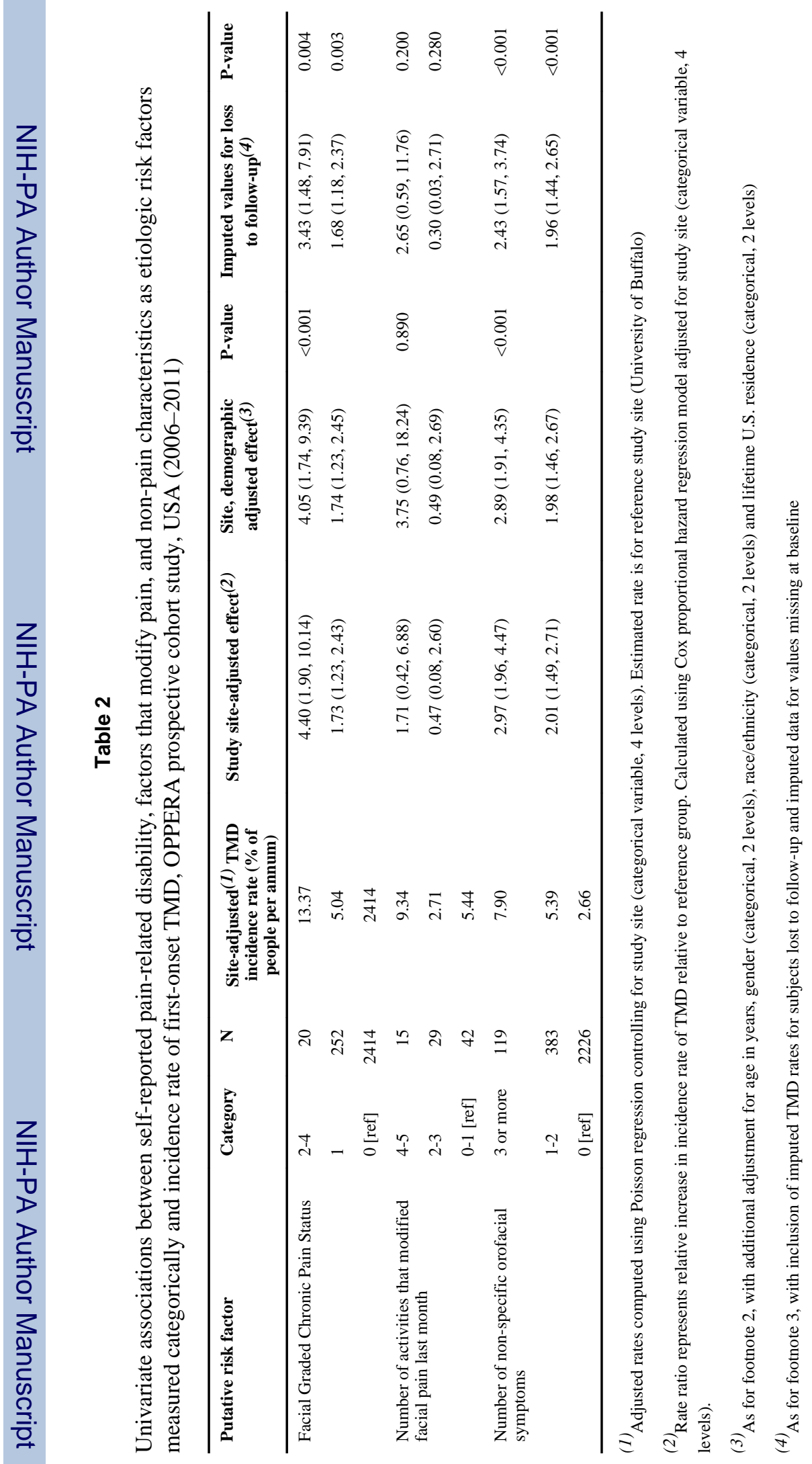




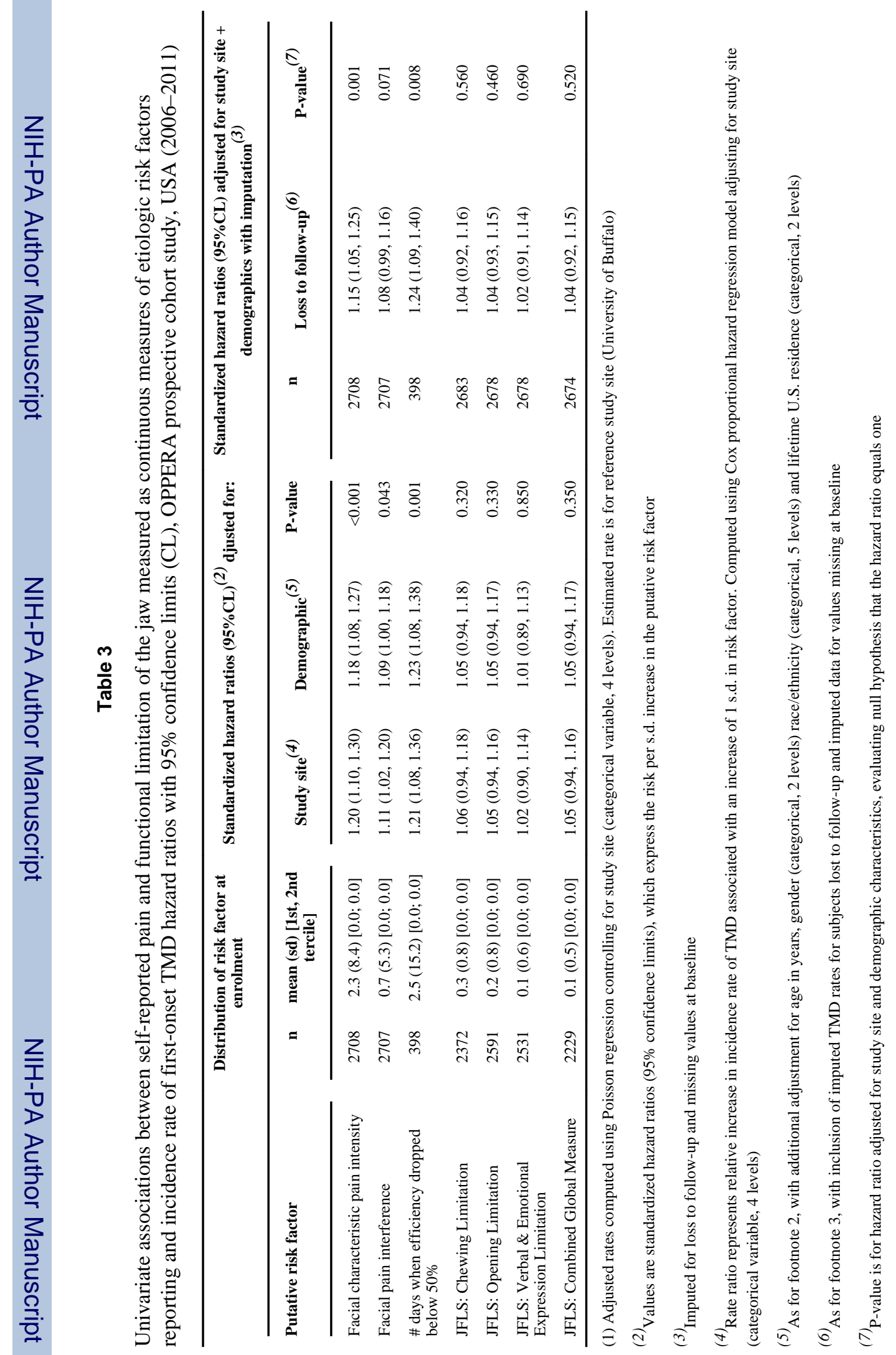




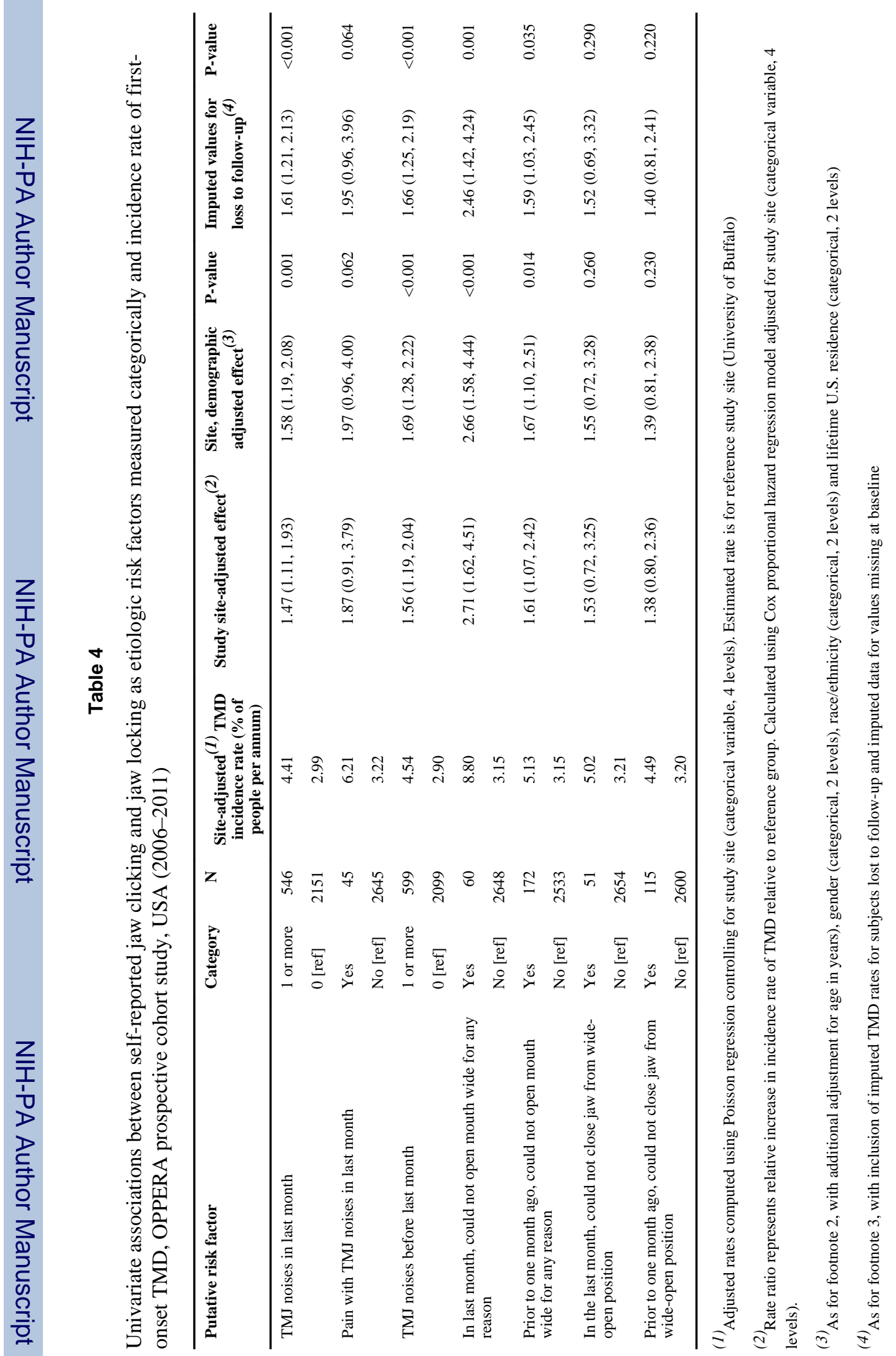

J Pain. Author manuscript; available in PMC 2014 December 01. 


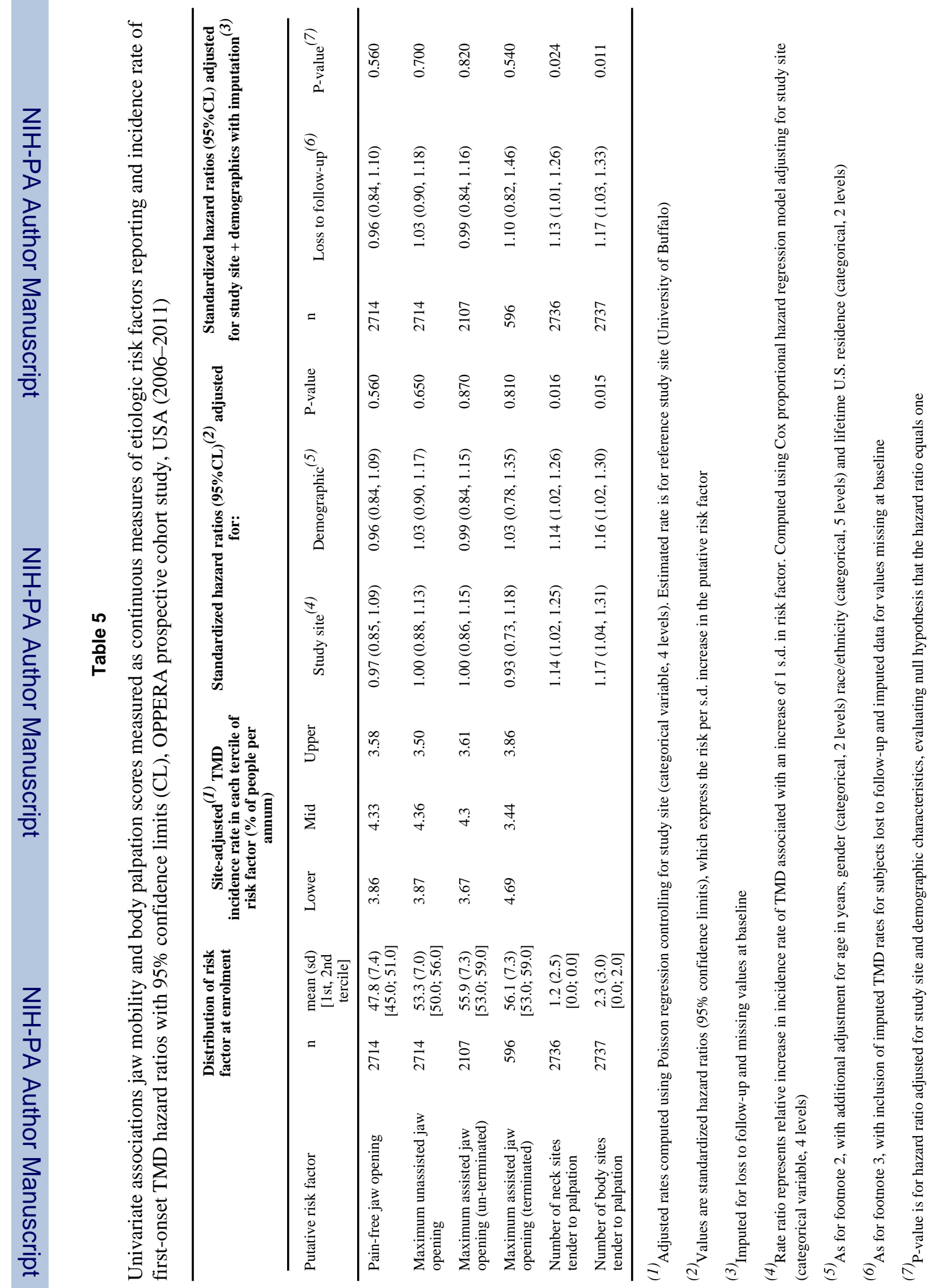

J Pain. Author manuscript; available in PMC 2014 December 01. 


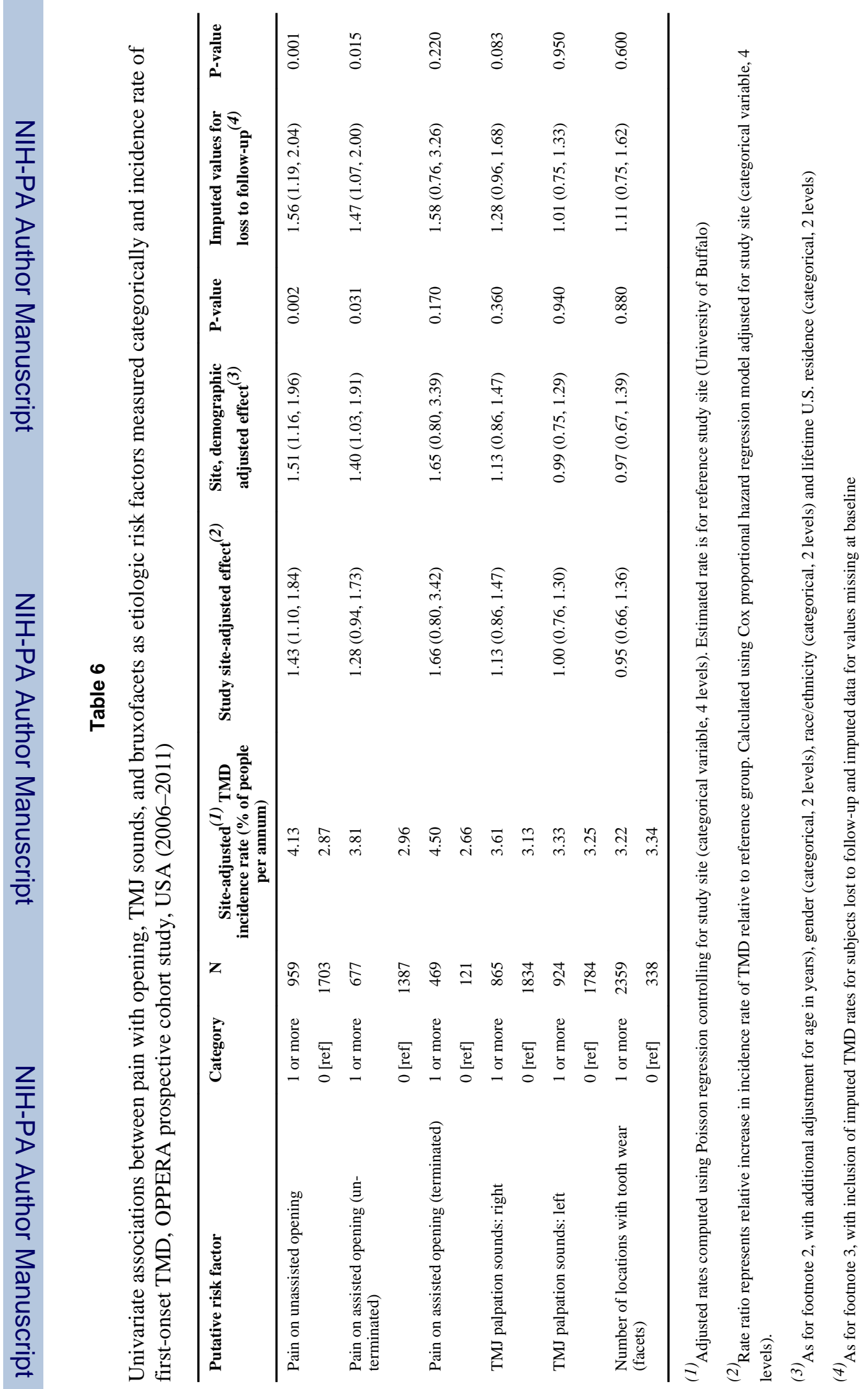

J Pain. Author manuscript; available in PMC 2014 December 01. 


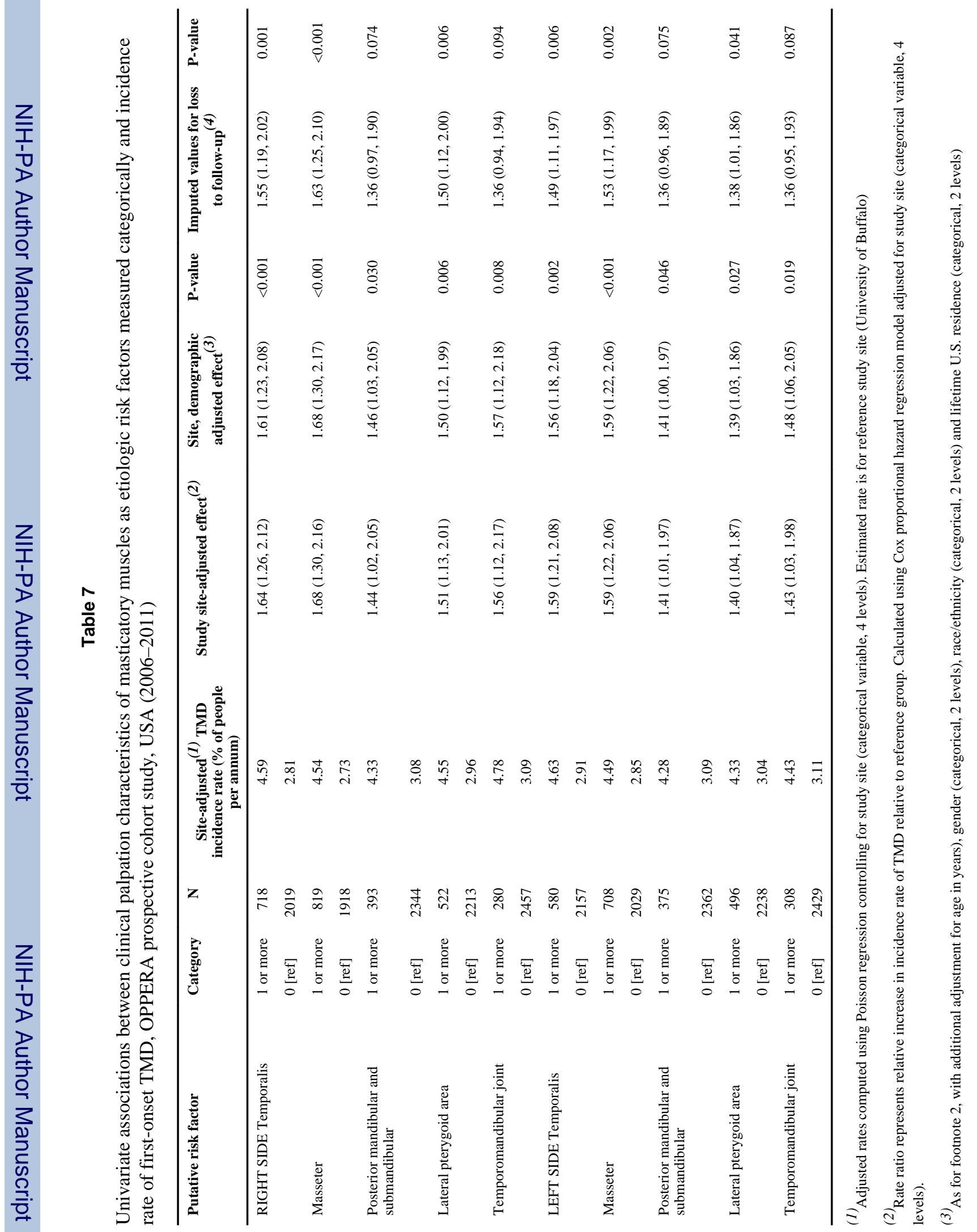




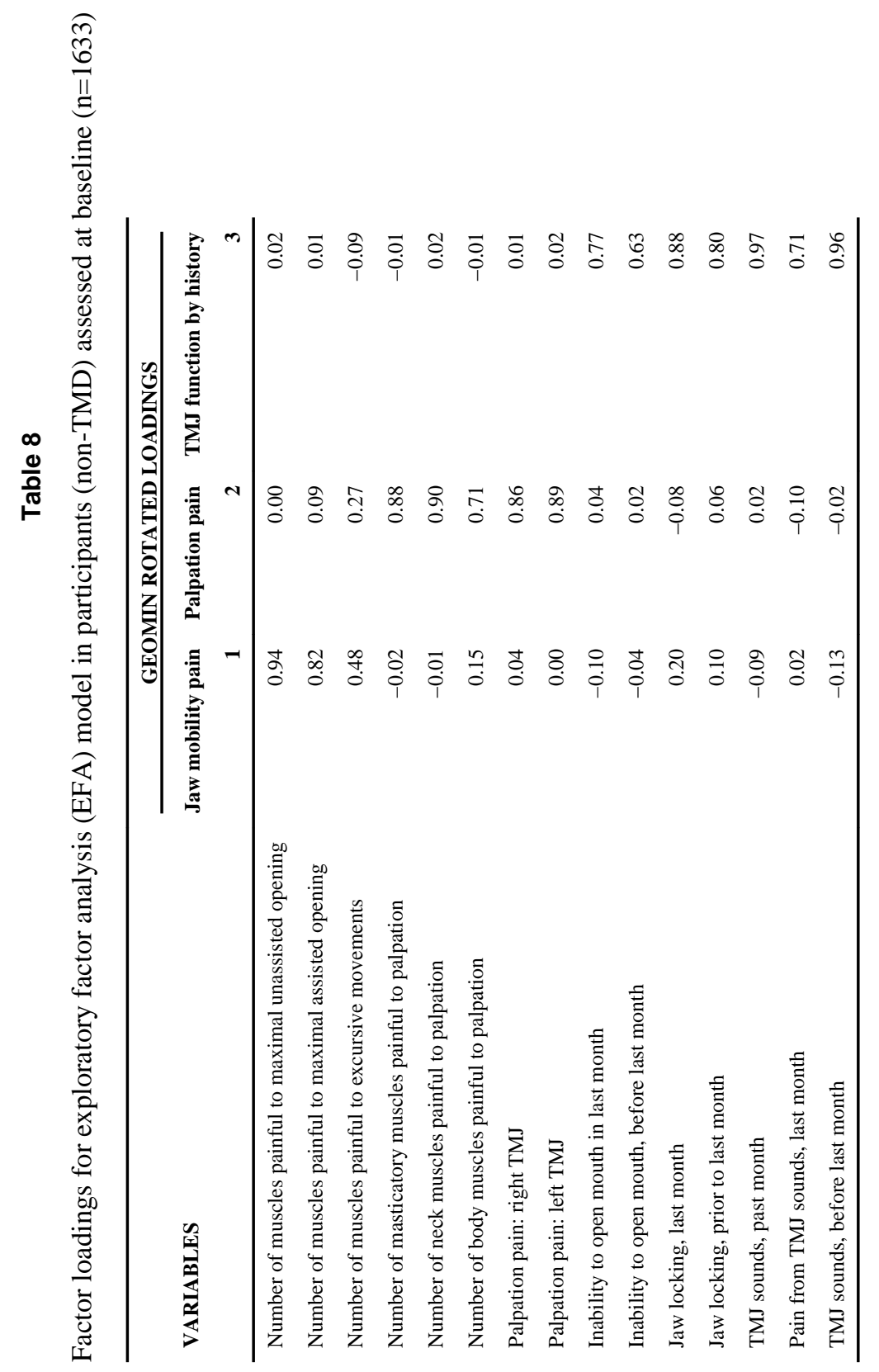

J Pain. Author manuscript; available in PMC 2014 December 01. 


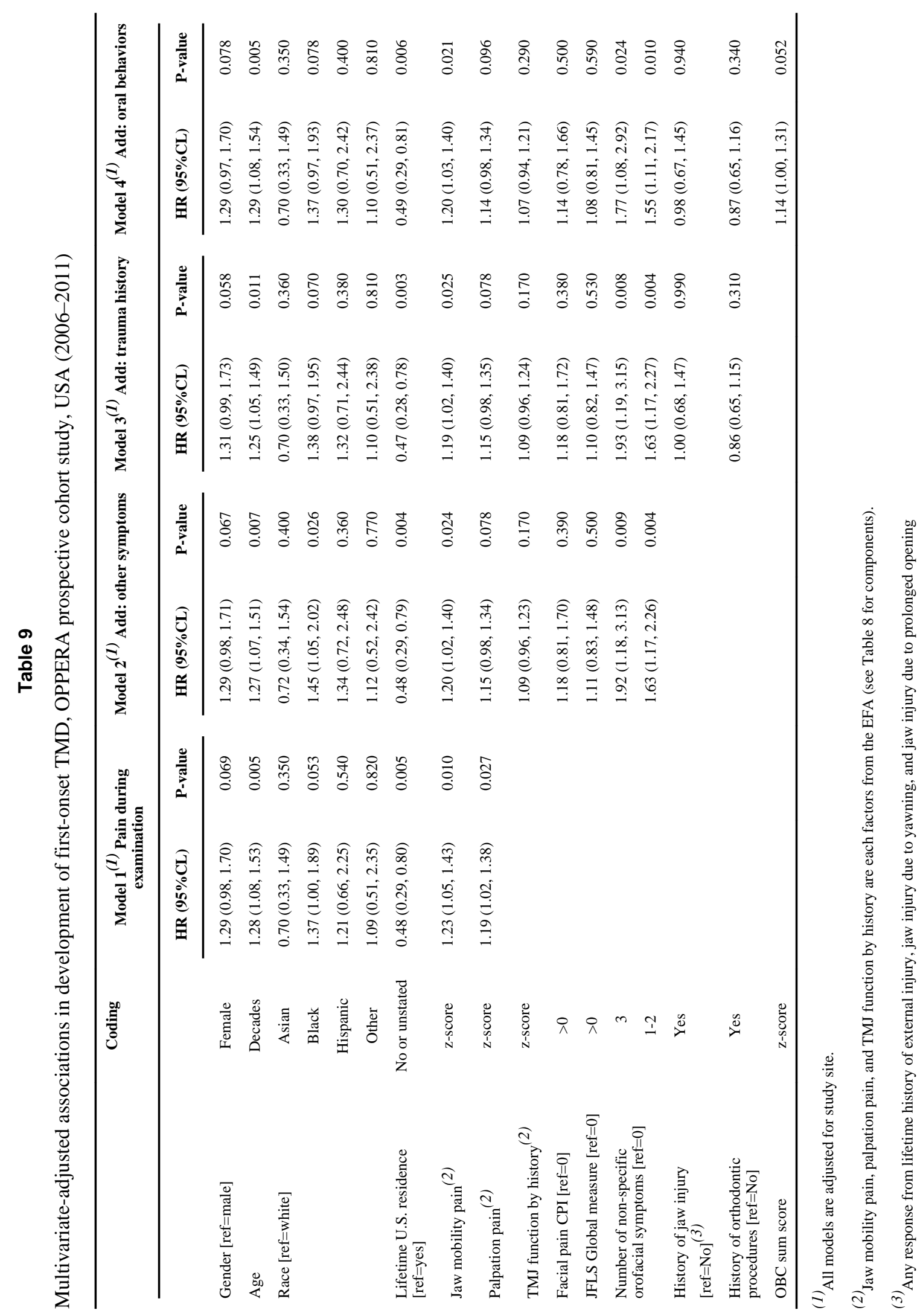

